\title{
One-loop electroweak effects on stop-chargino production at LHC
}

\author{
M. Beccaria ${ }^{a, b}$, G. Macorini ${ }^{c}$, E. Mirabella ${ }^{d}$, \\ L. Panizzi ${ }^{e, f}$, F.M. Renard ${ }^{g}$ and C. Verzegnassi ${ }^{e, f}$ \\ a Dipartimento di Fisica, Università del Salento, Italy \\ ${ }^{b}$ INFN, Sezione di Lecce, Italy \\ c ICTP, Trieste, Italy \\ ${ }^{d}$ Max-Planck-Institut für Physik (Werner Heisenberg Institut), Germany \\ e Dipartimento di Fisica Teorica, Università di Trieste, Italy \\ f INFN, Sezione di Trieste, Italy \\ $g$ Laboratoire de Physique Théorique et Astroparticules, \\ Université Montpellier II, France
}

\begin{abstract}
The process of stop-chargino production at LHC has been calculated in the Minimal Supersymmetric Standard Model at the complete electroweak one-loop level, assuming a mSUGRA symmetry breaking scheme. Several properties of the angular and invariant mass distributions of the basic $b g \rightarrow \tilde{t}_{a} \chi_{i}^{-}$amplitudes have been derived. For a meaningful collection of different benchmark points the overall electroweak one-loop effects are at most of the order of a few percent. At the realistically expected LHC accuracy, the main supersymmetric electroweak features of the process can be therefore essentially derived in this theoretical scheme from the simple Born level expressions.
\end{abstract}

PACS numbers: 


\section{INTRODUCTION}

The process of associated stop-chargino production at LHC has been recently considered as a potential source of information on SUSY parameters. In particular, it has been shown that the total rate would exhibit a possibly relevant dependence on $\tan \beta$ [1] and could also be sensitive to possible deviations from a Minimal Flavor Violation scheme [2]. In both cases, the calculations have been performed at the lowest electroweak order. SUSY QCD effects have been computed at NLO [3]. The conclusion was that these NLO strong supersymmetric effects in general enhance the LO total cross sections significantly, and thus must be carefully taken into account.

If Supersymmetry were discovered at LHC, and measurements of stop-chargino production began to be performed, the reasonable question would arise of whether the NLO electroweak supersymmetric effects might effectively change the special and relevant SUSY parameter dependence of the lowest order expressions given in Refs. [1, 2], in which case they should be also carefully taken into account, like the NLO QCD component. The aim of this paper is precisely that of performing an accurate calculation of the complete one-loop electroweak supersymmetric contributions to the stop-chargino production process. As a preliminary approach, we shall work in the Minimal Supersymmetric Standard Model, accept the validity of a mSUGRA symmetry breaking scheme and select a number of meaningful "benchmark" points to produce the final numerical predictions.

Technically speaking, the paper is organized as follows. Sect II will be devoted to a descrip-

tion of the shape and of the basic properties of the parton level amplitudes for $b g \rightarrow \tilde{t}_{a} \chi_{i}^{-}$ at Born and at one-loop level. A detailed analysis at Born level of the dependence of the total rate on supersymmetric parameters will be performed. Illustrations will be given for the angular distributions and for the invariant mass dependence of the helicity amplitudes near threshold and in the high energy range, where we have checked the agreement with the logarithmic terms of the Sudakov expansion. Sect III will exhibit the numerical one-loop effects on the production rates for a selected number of typical SUSY benchmark points. As a general feature, the effects will turn out to be numerically small, of a relative few percent at most, which would hardly be effective at the realistically expected LHC experimental accuracy. 


\section{KINEMATICS AND AMPLITUDES OF THE PROCESS $b g \rightarrow \tilde{t}_{a} \chi_{i}^{-}$}

The kinematics of the process $b g \rightarrow \tilde{t}_{a} \chi_{i}^{-}$is expressed in terms of the Dirac spinors $u\left(p_{b}, \lambda_{b}\right)$ and $\bar{v}_{c}\left(p_{\chi_{i}^{-}}, \lambda_{\chi_{i}^{-}}\right)$with the momenta:

$$
\begin{gathered}
p_{b}=\left(E_{b} ; 0,0, p\right) \quad p_{\tilde{t}_{a}}=\left(E_{\tilde{t}_{a}} ; p^{\prime} \sin \theta, 0, p^{\prime} \cos \theta\right) \\
p_{g}=(p ; 0,0,-p) \quad p_{\chi_{i}^{-}}=\left(E_{\chi_{i}^{-}} ;-p^{\prime} \sin \theta, 0,-p^{\prime} \cos \theta\right)
\end{gathered}
$$

and the gluon polarization vector:

$$
e_{g}\left(\lambda_{g}\right)=\left(0 ; \frac{\lambda_{g}}{\sqrt{2}},-\frac{i}{\sqrt{2}}, 0\right)
$$

referring to the helicity labels $\lambda_{b}= \pm 1 / 2, \lambda_{g}= \pm 1, \lambda_{\chi_{i}^{-}}= \pm 1 / 2$.

The angle $\theta$ refers to $p_{\tilde{t}_{a}}$ and $p_{b}$. We will use $s=\left(p_{b}+p_{g}\right)^{2}, t=\left(p_{b}-p_{\tilde{t}_{a}}\right)^{2}$ and $u=\left(p_{b}-p_{\chi_{i}^{-}}\right)^{2}$. The top squark states $\tilde{t}_{a}(a=1,2)$ are mixed states of $\tilde{t}_{L, R}$ with an angle $\theta_{t}$ and the chargino states $\chi_{i}^{-}(i=1,2)$ are mixed states of gauginos and Higgsinos with matrix elements $Z_{i j}^{ \pm}$. The process will be described by 8 helicity amplitudes $F_{\lambda_{b}, \lambda_{g}, \lambda_{\chi_{i}^{-}}}$related to the 8 invariant amplitudes $(k=1,4$ and $\eta=R, L)$ :

$$
\begin{gathered}
A=\left(\frac{\lambda}{2}\right)_{b, \tilde{t}_{a}} \sum_{k, \eta}\left[\bar{v}_{c}\left(p_{\chi_{i}^{-}}\right) J_{k \eta} u\left(p_{b}, \lambda_{b}\right)\right] N_{k \eta}(s, t, u) \\
J_{1 \eta}=\not p_{g} \notin P_{\eta} \quad J_{2 \eta}=\left(e \cdot p_{\chi}\right) P_{\eta} \\
J_{3 \eta}=\phi P_{\eta} \quad J_{4 \eta}=\left(e \cdot p_{\chi}\right) \not p_{g} P_{\eta}
\end{gathered}
$$

with $P_{\eta}=P_{R, L}=\left(1 \pm \gamma^{5}\right) / 2$. A colour matrix element $\left(\frac{\lambda}{2}\right)$ relating the initial $b$ quark and the final $\tilde{t}_{a}$ squark has been systematically factorized out.

Averaging over initial spins and colours and summing over final spins and colours with:

$$
\sum_{c o l}<\frac{\lambda^{l}}{2}><\frac{\lambda^{l}}{2}>=4
$$

leads to the elementary cross section:

$$
\frac{d \sigma}{d \cos \theta}=\frac{\beta^{\prime}}{768 \pi s \beta} \sum_{\text {spins }}\left|F_{\lambda_{b}, \lambda_{g}, \lambda_{\chi_{i}^{-}}}\right|^{2}
$$

where $\beta=2 p / \sqrt{s}, \beta^{\prime}=2 p^{\prime} / \sqrt{s}$.

The 8 scalar functions $N_{k \eta}(s, t, u)$ are obtained in terms of Born and one-loop diagrams. The Born terms result from the s-channel b exchange and the u-channel $\tilde{t}_{a}$ exchange:

$$
N_{1 \eta}^{\text {Born } s}=-g_{s} \frac{A_{i}^{\eta}\left(\tilde{t}_{a}\right)}{s-m_{b}^{2}}
$$




$$
N_{2 \eta}^{\text {Born } u}=2 g_{s} \frac{A_{i}^{\eta}\left(\tilde{t}_{a}\right)}{u-m_{\tilde{t}_{a}}^{2}}
$$

with the couplings:

$$
A_{i}^{L}\left(\tilde{t}_{L}\right)=-\frac{e}{s_{W}} Z_{1 i}^{+} \quad A_{i}^{L}\left(\tilde{t}_{R}\right)=\frac{e m_{t}}{\sqrt{2} M_{W} s_{W} \sin \beta} Z_{2 i}^{+} \quad A_{i}^{R}\left(\tilde{t}_{L}\right)=\frac{e m_{b}}{\sqrt{2} M_{W} s_{W} \cos \beta} Z_{2 i}^{-*}
$$

Using the Dirac decomposition explicitly given in App. A of [1] one gets the Born contribution to the 8 helicity amplitudes. Let us notice their basic properties which will be essential to understand the final results. First, because of the small value of $m_{b}$, the $b$ helicity corresponds to the chirality $\eta$ ( $\mathrm{L}$ for $\lambda_{b}=-1 / 2$ and $\mathrm{R}$ for $\lambda_{b}=+1 / 2$ ). In the case of the production of the lightest chargino $(i=1)$ this means that the $\lambda_{b}=-1 / 2$ amplitudes will generally dominate because the R chirality couplings (see eq.(11)) are depressed by the $m_{b}$ factor and by the non-diagonal chargino mixing element $Z_{2 i}^{-*}$. One can then predict the main features of the angular and of the energy dependences using again App. A of [1]. At low energy (near above threshold) the $\mathrm{u}$-channel contribution is suppressed by the final momentum $p^{\prime}$. Only the s-channel contribution survives and the leading amplitudes should be $F_{--+}$and $F_{---}$. They respectively produce an angular distribution $(1-\cos \theta)$ and $(1+\cos \theta)$. Having the same magnitude (at Born level) the unpolarized cross section should then be flat.

At high energy $(\sqrt{s} \gg m)$ one observes a cancellation between the s-channel and the $\mathrm{u}$ channel Born contributions to $F_{+++}, F_{++-}, F_{---}, F_{--+}$(see App.A of [1]), as well as the mass suppression of the $\mathrm{u}$-channel contribution to $F_{+-+}, F_{-+-}$(because $r_{b}$ and $r_{\chi}$ tend to 1). The only surviving amplitudes at high energy are then $F_{+--}$and $F_{-++}$:

$$
F_{+--}^{\text {Born } u} \rightarrow g_{s} \sqrt{2} A_{i}^{R}\left(\tilde{t}_{a}\right) \sin \frac{\theta}{2} \quad F_{-++}^{\text {Born } u} \rightarrow-g_{s} \sqrt{2} A_{i}^{L}\left(\tilde{t}_{a}\right) \sin \frac{\theta}{2}
$$

In this high energy limit the quantities of Eq.(12) can be expressed in terms of 3 basic amplitudes, one of gaugino type $F_{-++}\left(\tilde{t}_{L}\right)$ and two of higgsino type $F_{-++}\left(\tilde{t}_{R}\right), F_{+--}\left(\tilde{t}_{L}\right)$. In all cases the high energy distribution should tend to a $(1-\cos \theta)$ shape. For $\chi_{1}$ production and for the reasons already given above, $F_{-++}$should dominate. For a light stop $\tilde{t}_{1}$, mixture of $\tilde{t}_{L}$ and $\tilde{t}_{R}$, this amplitude will be:

$$
\cos \theta_{t} F_{-++}\left(\tilde{t}_{L}\right)+\sin \theta_{t} F_{-++}\left(\tilde{t}_{R}\right)
$$




\section{A. Parameter Dependence at Born Level}

Remaining at Born level it is already possible to extract relevant e.w. information from the process. Although some preliminary search of this kind already exists [1, 2], we will devote this Section to a brief updated summary of the main information that could be derived from this approximate treatment.

Indeed, at Born level a limited set of parameters affects the determination of physical observables. Besides the values of the stop and chargino masses, which are obviously crucial for the definition of the production threshold, other SUSY parameters contribute to the the coupling $b \tilde{t}_{a} \chi_{i}^{-}$, Eq.(11). While $\tan \beta$ explicitly appears in the various terms, the chargino mixing matrices $Z_{i j}^{ \pm}$depend in a non-trivial way on parameters of the chargino mass matrix:

$$
X=\left(\begin{array}{cc}
M_{2} & \sqrt{2} M_{W} \sin \beta \\
\sqrt{2} M_{W} \cos \beta & \mu
\end{array}\right)
$$

Moreover, for production of physical stops, the mixing angle $\theta_{\tilde{t}}$ mixes the various terms of Eq.(11). In conclusion, it is possible to identify a set of independent parameters which determine the amplitude at Born level:

$$
\tan \beta \quad M_{2} \quad \mu \quad m_{\tilde{t}} \quad \theta_{\tilde{t}}
$$

The chargino masses are determined by a combination of $M_{2}, \mu$ and $\tan \beta$. Since to perform a parameter analysis of the process it seems reasonable to fix all the masses, it is possible to trade, e.g., $M_{2}$ for $m_{\chi_{1}}$ and $\mu$ for $m_{\chi_{2}}$, depending on the chargino of the final state.

Given these premises, the process of production of the lightest stop and chargino $b g \rightarrow \tilde{t}_{1} \chi_{1}$ will now be analyzed at Born level to investigate possible dependences on supersymmetric parameters. Rather than looking for a dependence of the cross section on the stop or chargino masses that we assumed to be experimentally known from previous discovery, we looked for dependences on $\tan \beta, \mu$ and $\theta_{\tilde{t}}$. The results of the analysis are shown in Fig. 1 All panels show the dependence of the total cross section on the mixing angle $\theta_{\tilde{t}}$ for different values of $\tan \beta$, at different values of $\mu$. In particular, Figs. 1(a) and 1(b) show the results obtained for low values of $\mu$, close to the chargino mass, while in Figs. 1(c) and $1(\mathrm{~d}) \mu$ has been pushed to the value of $800 \mathrm{Gev}$, which is high compared to $m_{\chi_{1}}$. It is possible to notice that the cross section depends very strongly on the value of $\theta_{\tilde{t}}$ and that there is always a value of the angle for which the cross section drops near to zero. In Fig. 1(a), where the low 
threshold allows a cross section of the order of the pb, it is possible to see that $\sigma$ changes from $\sim 6 \mathrm{pb}$ for $\theta_{\tilde{t}} \simeq \pi / 8$ to less than $0.5 \mathrm{pb}$ for $\theta_{\tilde{t}} \simeq 5 \pi / 8$ (when $\tan \beta=40$ ). Therefore there are regions of the parameter space where, even if the masses of final state particles are very low, the stop mixing angle pushes the cross section to nearly undetectable levels. The dependence on $\theta_{\tilde{t}}$ can indeed be understood looking at the amplitude, which is a sum of terms of the form:

$$
A_{1} \cos \theta_{\tilde{t}}+A_{2} \sin \theta_{\tilde{t}}
$$

Depending on the values of $A_{1}$ and $A_{2}$, the squared amplitude generates the curves shown in Fig. 1.

The results also depend in a weaker and less trivial way on $\tan \beta$. The cross section is pushed to somewhat higher values as $\tan \beta$ increases, but to different extents in the various considered cases: the variation of the total cross sections with different values of $\tan \beta$ are strongly affected by the choice of $\mu$ resulting in a very mild dependence on $\tan \beta$ for high values of $\mu$ and viceversa, so that the determination of this supersymmetric parameter from this process could be ambiguous, unless the rate turns out to be larger than a certain "threshold" value. Further constraints coming from other processes could however limit the range of $\tan \beta$, and the determination of the two remaining parameters through the analysis of this process would then be relevant.

\section{B. One-Loop Amplitude}

For the calculation of the one-loop amplitude we use the on-shell scheme; the one-loop electroweak terms can be classified in:

- counter terms for $b, \tilde{t}_{a}, \chi_{i}^{-}$lines, coupling constants and mixing elements, all of them being expressed in terms of self-energy diagrams;

— self-energy corrections for $b$ and $\tilde{t}_{a}$ propagators;

— s-channel left and right triangles;

- u-channels bubbles with 4-leg couplings and up, down triangles;

— direct boxes, crossed boxes, twisted boxes; 
and the related diagrams are shown in Figs. 2,5.

Since the complete expressions of the various conter-terms and self energies are rather involved we list them separately in the App. A. All the contributions of the counter-terms and self energies, together with the virtual vertex and box diagrams have been computed using the usual decomposition in terms of Passarino-Veltman functions and the complete amplitude has been implemented in the numerical code TigreMC.

We have checked the cancellation of the UV divergences among counter terms, selfenergies and triangles, this cancellation occuring separately for s-channel and for u-channel, as well as for gauge-left, gauge-right, Yukawa-left and Yukawa-right sectors separately.

Another useful check can be done using the high energy behaviour of the amplitudes. High energy rules [4] predict the logarithmic behaviour of these amplitudes at one-loop level. They use splitting functions for external particles $b, \tilde{t}_{L, R}, \chi_{i}^{-}$and Renormalization Group effects on the parameters appearing in the Born terms. They read:

$$
\begin{aligned}
F_{-++}\left(\tilde{t}_{L}\right)=-g_{s} \sqrt{2} A_{i}^{L}\left(\tilde{t}_{L}\right) \sin \frac{\theta}{2}\left\{1+\frac{\alpha}{4 \pi}\{\right. \\
\frac{1+26 c_{W}^{2}}{18 s_{W}^{2} c_{W}^{2}} \log \frac{s}{M_{W}^{2}}-\left[\frac{m_{t}^{2}}{2 s_{W}^{2} M_{W}^{2}}\left(1+\cot ^{2} \beta\right)+\frac{m_{b}^{2}}{2 s_{W}^{2} M_{W}^{2}}\left(1+\tan ^{2} \beta\right)\right] \log \frac{s}{M_{W}^{2}} \\
\left.\left.-\left\{\frac{1}{2 s_{W}^{2}} \log ^{2} \frac{-u}{m_{W}^{2}}+\log ^{2} \frac{-u}{m_{Z}^{2}}\right]+\frac{1-10 c_{W}^{2}}{36 s_{W}^{2} c_{W}^{2}} \log ^{2} \frac{-t}{m_{Z}^{2}}\right\}\right\} \\
F_{-++}\left(\tilde{t}_{R}\right)=-g_{s} \sqrt{2} A_{i}^{L}\left(\tilde{t}_{R}\right) \sin \frac{\theta}{2}\left\{1+\frac{\alpha}{4 \pi} .\right. \\
\left\{-\left[\frac{1}{3 c_{W}^{2}}\right] \log ^{2} \frac{s}{m_{Z}^{2}}-\left[\frac{1}{9 c_{W}^{2}}\right] \log ^{2} \frac{-t}{m_{W}^{2}}\right. \\
\left.\left.+\frac{1-4 c_{W}^{2}}{12 s_{W}^{2} c_{W}^{2}}\left[\log ^{2} \frac{-u}{M_{Z}^{2}}\right]-\frac{1}{2 s_{W}^{2}}\left[\log ^{2} \frac{-u}{M_{W}^{2}}\right]\right\}\right\} \\
F_{+--}\left(\tilde{t}_{L}\right)= \\
g_{s} \sqrt{2} A_{i}^{R}\left(\tilde{t}_{L}\right) \sin _{\frac{\theta}{2}}\left\{1+\frac{\alpha}{4 \pi} \cdot\right. \\
\left\{-\left[\frac{1+2 c_{W}^{2}}{12 s_{W}^{2} c_{W}^{2}}\right] \log ^{2} \frac{s}{m_{Z}^{2}}-\left[\frac{1}{2 s_{W}^{2}}\right] \log ^{2} \frac{s}{m_{W}^{2}}\right. \\
\left.\left.+\frac{1}{18 c_{W}^{2}}\left[\log ^{2} \frac{-t}{M_{W}^{2}}\right]-\frac{1}{6 s_{W}^{2}}\left[\log ^{2} \frac{-u}{M_{W}^{2}}\right]\right\}\right\}
\end{aligned}
$$

The logarithmic part of the gaugino amplitude (17) is similar to the one obtained for the process $b g \rightarrow t W^{-}$with transverse $W[5]$. Higgsino amplitudes $F_{-++}\left(\tilde{t}_{R}\right), F_{+--}\left(\tilde{t}_{L}\right)$ in 
(18,19) get logarithmic terms similar to the ones in both $b g \rightarrow t W^{-}$for longitudinal $W$ and $b g \rightarrow t H^{-}[6]$. One notices that there is no linear logarithmic contribution , but only quadratic logarithmic terms, in these Higgs or Higgsino type of amplitudes. The coefficients of these quadratic logarithms are of pure gauge origin and do not involve any free parameter.

Taking our complete one-loop computation and retaining only the logarithmic parts of the B,C,D Passarino-Veltman functions appearing in the various diagrams, we do recover the above expressions for the 3 types of leading amplitudes.

We now give illustrations of the various features mentioned above for the process $b g \rightarrow \tilde{t_{1}} \chi_{1}$ with production of the lightest stop and chargino. We choose two typical benchmark MSSM points called LS1 and LS2 whose characteristics are shown in Tab! together with those of all the benchmark points that we have used for the analysis (see next Section for more details).

\begin{tabular}{|c|ccccc||c|c|}
\hline mSUGRA scenario & $m_{0}$ & $m_{1 / 2}$ & $A_{0}$ & $\tan \beta$ & $\operatorname{sign} \mu$ & $m_{\widetilde{t}_{1}}$ & $m_{\chi_{1}}$ \\
\hline LS1 & 300 & 150 & -500 & 10 & + & 214.6 & 103.6 \\
LS2 & 300 & 150 & -500 & 50 & + & 224.7 & 106.9 \\
SPS5 & 150 & 300 & -1000 & 5 & + & 279.0 & 226.2 \\
SU1 & 70 & 350 & 0 & 10 & + & 566.4 & 255.7 \\
SU6 & 320 & 375 & 0 & 50 & + & 634.1 & 279.7 \\
\hline
\end{tabular}

TABLE I: mSUGRA benchmark points and masses of the lightest stop and chargino (all the values are in $\mathrm{GeV}$ )

Fig. 6] shows the energy dependence of each helicity amplitude from threshold to high energy for a given (central) angle $\theta=\pi / 4$. For each amplitude two curves represent the Born and the full one loop result. One can check that they confirm the expectations described in the previous subsection, namely the nature of:

— the dominant amplitudes at low energy

— the dominant amplitudes at high energy 
The size of the one-loop correction is of the order of few percent, and one sees that the high energy behaviour is quickly reached as soon as the threshold is crossed.

The difference between the LS1 and LS2 cases is due to the increase in the final masses and in the change in the stop and in the chargino mixings. In particular for LS2 the R chirality amplitudes are less depressed because of the the difference in $\tan \beta$ and in the

mixing element $Z_{2 i}^{-}$, which increase the value of $A_{i}^{R}\left(\tilde{t}_{L}\right)$ (see Eq.(11) ) and this can be clearly seen both at low and at high energies.

Fig. 7 gives the angular distributions at low energy $\left(s / s_{\text {thresh. }}=1.001\right)$ and at high energy $\left(s / s_{\text {thresh. }}=30\right)$. At low energy the leading amplitudes give indeed the expected $(1+\cos \theta)$ and $(1-\cos \theta)$ distributions, whereas at high energy one tends to a limiting $(1-\cos \theta)$ distribution, at least away from purely backward scattering. The one-loop corrections make only little changes in the shape of the angular distributions as expected from the Sudakov rules.

\section{QED radiation}

The $\mathcal{O}(\alpha)$ electroweak corrections include contributions from virtual and from real photon emission. The virtual photon exchange diagrams belong to the complete set of electroweak virtual corrections, and are necessary for the gauge invariance of the final result. The singularities associated with the massless nature of the photon have been regularized by introducing a small photon mass $m_{\gamma}$. The real radiation contribution has been split into a soft part, derived within the eikonal approximation, where the photon energy has been integrated from the lower bound $m_{\gamma}$ to a maximum cut-off $\Delta E$, and into a hard part, integrated from the minimum photon energy $\Delta E$ to the maximum allowed kinematical value. The soft real contribution contains explicitly the photon mass parameter $m_{\gamma}$ while the hard part can be calculated with a massless external photon. The complete matrix element for real radiation, including fermion mass effects, has been calculated analytically with the help of FeynArts [7] and FormCalc [8].

The logarithmic terms containing $m_{\gamma}$ cancel exactly in the sum of virtual and soft real part, leaving only polynomial spurious terms, which approach zero at least as $m_{\gamma}^{2}$. We have numerically checked the cancellation by taking the limit $m_{\gamma} \rightarrow 0$ of our computation. The 
large collinear logarithms containing the bottom mass are only partially cancelled when real and virtual corrections are summed together, but they can be absorbed into the definition of the parton distribution functions (PDFs). This can be achieved redefining the bottom PDF according to a factorization scheme. In the $\overline{\mathrm{MS}}$ (DIS) scheme such redefinition reads [9]

$$
\begin{aligned}
b(x, \mu) & \rightarrow b(x, \mu)\left\{1-\frac{\alpha}{\pi} e_{b}^{2}\left[1-\ln \delta_{s}-\ln \delta_{s}^{2}+\left(\ln \delta_{s}+\frac{3}{4}\right) \ln \left(\frac{\mu^{2}}{m_{b}^{2}}\right)-\frac{1}{4} \lambda_{\mathrm{FC}} f_{1}\right]\right\} \\
& -\frac{\alpha}{2 \pi} e_{b}^{2} \int_{x}^{1-\delta_{s}} \frac{d z}{z} b\left(\frac{x}{z}, \mu\right)\left[\frac{1+z^{2}}{1-z} \ln \left(\frac{\mu^{2}}{m_{b}^{2}} \frac{1}{(1-z)^{2}}\right)-\frac{1+z^{2}}{1-z}+\lambda_{\mathrm{FC}} f_{2}\right],
\end{aligned}
$$

with $\lambda_{\mathrm{FC}}=0\left(\lambda_{\mathrm{FC}}=1\right) . \mu$ is the factorization scale, $\delta_{s}=2 \Delta E / \sqrt{s}$, while $e_{b}$ is the bottom charge. $f_{1}$ and $f_{2}$ are defined as follows,

$$
\begin{aligned}
& f_{1}=9+\frac{2}{3} \pi^{2}+3 \ln \delta_{s}-2 \ln ^{2} \delta_{s} \\
& f_{2}=\frac{1+z^{2}}{1-z} \ln \left(\frac{1-z}{z}\right)-\frac{3}{2} \frac{1}{1-z}+2 z+3
\end{aligned}
$$

The calculation of the full $\mathcal{O}(\alpha)$ corrections to any hadronic observable must include QED effects in the DGLAP evolution equations. Such effects are taken into account in the MRST2004QED PDF [10]. This set is however NLO QCD, while our computation is leading order QCD. Therefore, analogously to [11], a LO QCD PDF set has been chosen, namely the CTEQ6L [12]. This choice is justified by the fact that QED effects are known to be small [13]. In the numerical analyses we have used the $\overline{\mathrm{MS}}$ factorization scheme at the scale $\mu=\left(m_{\tilde{t}_{1}}+m_{\tilde{\chi}_{1}^{-}}\right)$. It is worth to mention that the dependence of the full $\mathcal{O}(\alpha)$ contribution on the factorization scheme is rather weak. Indeed, if the DIS factorization scheme is used instead of $\overline{\mathrm{MS}}$, the differences in the numerical value of the one-loop electroweak effects are of the order of $0.01 \%$ in all the considered mSUGRA benchmark points.

The final cross section has to be independent of the fictitious separator $\Delta E$, for sufficiently small $\Delta E$ values. This has been checked numerically to hold for $\Delta E \leq 1 \mathrm{GeV}$, as shown in Figure 8 (lower panel), despite the strong sensitivity to $\Delta E$ of the soft plus virtual and of the hard cross section separately, as shown in Figure 8 (upper panel).

Similarly to what has been obtained in our previous works [14] and [15], QED contributions to the total cross section are positive with a relative size of the order of a few percent. 


\section{ONE-LOOP RESULTS}

The distribution of the invariant mass of the final states $d \sigma / d M_{i n v}$ has been evaluated at the one-loop electroweak level for a number of SUSY benchmark points (assuming a mSUGRA supersymmetry breaking) with a wide variation of mass spectra. The obtained cross sections at the Born and one-loop level for five representative points (the "Light SUSY" LS1, LS2, discussed in [16], the ATLAS SU1 and SU6 [17] and the SPS5 "Light Stop scenario" [18]) are collected in Tab. II: for the realistic case of production of the lightest stop and chargino states $\tilde{t}_{1}$ and $\chi_{1}^{-}$, only the couple LS1 - LS2 give a cross section of order of the $p b$ (considering a global factor 2, arising from the conjugate process), that we shall consider in this paper as a reasonable limit for realistic detections at the LHC. All the other input sets, including the SPS5 "Light Stop", give smaller rates, and will not be further considered in what follows.

For what concerns the one-loop electroweak corrections, we have found that they are generally small, of the order of a relative few percent for all the considered scenarios. As

\begin{tabular}{|c|ccc|}
\hline mSUGRA scenario & $\sigma_{\text {Born }}$ & $\sigma_{1-\text { loop }}$ & \% Effect \\
\hline LS1 & 0.4287 & 0.4442 & 3.6 \\
LS2 & 0.5419 & 0.5436 & 0.3 \\
SPS5 & 0.05704 & 0.05810 & 1.8 \\
SU1 & 0.004052 & 0.004041 & -0.3 \\
SU6 & 0.002541 & 0.002576 & 1.4 \\
\hline
\end{tabular}

TABLE II: Total cross section at Born and loop level for the five considered benchmark points

an example of this behaviour in Figs. 9 and 10 we plot the differential distibutions for the LS1 and LS2 benchmark points, (the points with highest cross sections): as one can notice, the one-loop effect is positive in the low energy region (near the production threshold) and drops to negative values increasing the final invariant mass. The global effect on the totally integrated cross section, being the result of the sum of two opposite contributions, is positive $(3.6 \%)$ in the LS1 case, slightly smaller and below the 1\% in the LS2 case.

The conclusion of our analysis is thus, for what concerns the possibility that NLO electroweak effects might affect the stop-chargino production process, essentially negative in 
the chosen theoretical scheme, given the fact that a realistic experimental accuracy of the measurements of the various rates should hardly be better than, say, ten percent or more

([19]). In this spirit, it appears that the complete dependence on the SUSY parameters can be satisfactorily provided by the simple Born expressions of the process discussed in the previous Section.

This conclusion is valid in the chosen theoretical scheme, and is based on the relative smallness of the one-loop electroweak effects. Clearly, the same conclusions cannot be drawn at this point for possible different supersymmetric schemes. As a personal feeling, it seems unlikely to us that strong one-loop effects might there arise, simply given the unavoidably large sizes of the virtually exchanged sparticles. However, if LHC discovered supersymmetry and reached a suitable experimental accuracy, an extended analysis of the process that we have considered might become definitely requested.

\section{CONCLUSIONS}

In this paper we have calculated the complete electroweak one-loop expression of the stop-chargino process in the MSSM assuming a mSUGRA symmetry breaking scheme, to evidentiate possible realistically "visible" effects. In our calculations we have verified the fulfillment of a number of theoretical requests, including the reproduction of asymptotic Sudakov expansions. This, we believe, should make our analysis reliable. As a result of our calculation we have concluded that the complete one-loop electroweak effect is of the relative few percent size, that would make it hardly visible in a realistic LHC situation. Given this result, the relevant e.w. information can be extracted from the Born expression of the rate. We have examined its possible dependence on those supersymmetric parameters, on which it depends, that cannot be directly measured from direct production, i.e. on the parameters $\mu, \tan \beta$ and $\theta_{\tilde{t}}$. Assuming a previous measurement of the stop and chargino masses, we have verified that the dependence of the rates on $\theta_{\tilde{t}}$ and $\mu$ might be rather strong in the case of light final state masses, and would influence the dependence on $\tan \beta$. This would indicate that, given a light stop and chargino masses picture, a measurement of the light stop-chargino process might provide an original and useful type of constraints on the size of the relevant MSSM parameters. 


\section{APPENDIX A: COUNTER TERMS}

The contributions to the s-channel of the counter terms terms are, symbolically:

$$
\begin{aligned}
N_{1 L}^{c . t . s}= & -\frac{g_{s}\left(\frac{\lambda^{l}}{2}\right)}{s-m_{b}^{2}}\left\{\frac{3}{2} \delta Z_{L}^{b} A_{i}^{L}\left(\tilde{t}_{a}\right)+\frac{1}{2} \sum_{a^{\prime}} \delta Z_{a^{\prime} a}^{*} A_{i}^{L}\left(\tilde{t}_{a^{\prime}}\right)\right. \\
& \left.+\delta A_{i}^{L}\left(\tilde{t}_{a}\right)+\frac{1}{2} \sum_{j} \delta \chi_{j i}^{L} A_{j}^{L}\left(\tilde{t}_{a}\right)\right\} \\
N_{1 R}^{c . t . s}= & -\frac{g_{s}\left(\frac{\lambda^{l}}{2}\right)}{s-m_{b}^{2}}\left\{\frac{3}{2} \delta Z_{R}^{b} A_{i}^{R}\left(\tilde{t}_{a}\right)+\frac{1}{2} \sum_{a^{\prime}} \delta Z_{a^{\prime} a}^{*} A_{i}^{R}\left(\tilde{t}_{a^{\prime}}\right)\right. \\
& \left.+\delta A_{i}^{R}\left(\tilde{t}_{a}\right)+\frac{1}{2} \sum_{j} \delta \chi_{j i}^{R} A_{j}^{R}\left(\tilde{t}_{a}\right)\right\} \\
N_{3 L}^{c . t . s}= & -\frac{m_{b} g_{s}\left(\frac{\lambda^{l}}{2}\right)}{s-m_{b}^{2}}\left\{\left(\delta Z_{L}^{b}+\frac{1}{2} \delta Z_{R}^{b}\right) A_{i}^{R}\left(\tilde{t}_{a}\right)+\frac{1}{2} \sum_{a^{\prime}} \delta Z_{a^{\prime} a}^{*} A_{i}^{R}\left(\tilde{t}_{a^{\prime}}\right)\right. \\
& \left.+\delta A_{i}^{R}\left(\tilde{t}_{a}\right)+\frac{1}{2} \sum_{j} \delta \chi_{j i}^{R} A_{j}^{R}\left(\tilde{t}_{a}\right)\right\}-m_{b} N_{1 R}^{c . t . s} \\
& -\frac{m_{b} g_{s}\left(\frac{\lambda^{l}}{2}\right)}{s-m_{b}^{2}}\left\{\left(\delta Z_{R}^{b}+\frac{1}{2} \delta Z_{L}^{b}\right) A_{i}^{L}\left(\tilde{t}_{a}\right)+\frac{1}{2} \sum_{a^{\prime}} \delta Z_{a^{\prime} a}^{*} A_{i}^{L}\left(\tilde{t}_{a^{\prime}}\right)\right. \\
& \left.+\delta A_{i}^{L}\left(\tilde{t}_{a}\right)+\frac{1}{2} \sum_{j} \delta \chi_{j i}^{L} A_{j}^{L}\left(\tilde{t}_{a}\right)\right\}-m_{b} N_{1 L}^{c . t . s} \\
N_{3 R}^{c . t . s}= &
\end{aligned}
$$

and from $b$ s.e. one gets $(\eta=+1,-1$ means $R, L)$ :

$$
\begin{aligned}
N_{1 \eta}^{\text {s.e. } s}= & g_{s}\left(\frac{\lambda^{l}}{2}\right) \frac{1}{\left(s-m_{b}^{2}\right)^{2}}\left[A _ { i } ^ { \eta } ( \tilde { t } _ { a } ) \left(s\left(\Sigma_{\eta}^{b}(s)+\delta Z_{\eta}^{b}\right)+m_{b}^{2}\left(\Sigma_{-\eta}^{b}(s)+\delta Z_{-\eta}^{b}\right)\right.\right. \\
& +2 m_{b}^{2}\left(\Sigma_{S}^{b}(s)-\frac{1}{2}\left(\delta Z_{\eta}^{b}+\delta Z_{-\eta}^{b}\right)-\frac{\delta m_{b}}{m_{b}}\right] \\
N_{3 \eta}^{\text {s.e. } s}=\quad & g_{s}\left(\frac{\lambda^{l}}{2}\right) \frac{1}{\left(s-m_{b}^{2}\right)^{2}}\left[A_{i}^{-\eta}\left(\tilde{t}_{a}\right)\left(s m_{b}\left(\Sigma_{\eta}^{b}(s)+\delta Z_{\eta}^{b}\right)+s m_{b}\left(\Sigma_{-\eta}^{b}(s)+\delta Z_{-\eta}^{b}\right)\right)\right. \\
+ & m_{b}\left(s+m_{b}^{2}\right)\left(\Sigma_{S}^{b}(s)-\frac{1}{2}\left(\delta Z_{\eta}^{b}+\delta Z_{-\eta}^{b}\right)-\frac{\delta m_{b}}{m_{b}}\right]-m_{b} N_{1-\eta}^{\text {s.e. } s}
\end{aligned}
$$

For the u-channel c.t. we obtain:

$$
\begin{aligned}
N_{2 L}^{c . t . u}= & 2 g_{s}\left(\frac{\lambda^{l}}{2}\right)\left\{\frac{1}{2} \delta Z_{L}^{b} A_{i}^{L}\left(\tilde{t}_{a}\right)\left(\frac{1}{u-m_{\tilde{t}_{a}}^{2}}\right)+\sum_{a^{\prime}} \bar{\delta} Z_{a^{\prime} a} A_{i}^{L}\left(\tilde{t}_{a^{\prime}}\right)\left(\frac{1}{u-m_{\tilde{t}_{a^{\prime}}}^{2}}\right)\right. \\
& +\frac{1}{2} \sum_{a^{\prime}} \delta Z_{a^{\prime} a}^{*} A_{i}^{L}\left(\tilde{t}_{a^{\prime}}\right)\left(\frac{1}{u-m_{\tilde{t}_{a}}^{2}}\right)+\delta A_{i}^{L}\left(\tilde{t}_{a}\right)\left(\frac{1}{u-m_{\tilde{t}_{a}}^{2}}\right) \\
& \left.+\frac{1}{2} \sum_{j} \delta \chi_{j i}^{L} A_{j}^{L}\left(\tilde{t}_{a}\right)\left(\frac{1}{u-m_{\tilde{t}_{a}}^{2}}\right)\right\}
\end{aligned}
$$




$$
\begin{aligned}
N_{2 R}^{c . t . u}= & 2 g_{s}\left(\frac{\lambda^{l}}{2}\right)\left\{\frac{1}{2} \delta Z_{R}^{b} A_{i}^{R}\left(\tilde{t}_{a}\right)\left(\frac{1}{u-m_{\tilde{t}_{a}}^{2}}\right)+\sum_{a^{\prime}} \bar{\delta} Z_{a^{\prime} a} A_{i}^{R}\left(\tilde{t}_{a^{\prime}}\right)\left(\frac{1}{u-m_{\tilde{t}_{a^{\prime}}}^{2}}\right)\right. \\
& +\frac{1}{2} \sum_{a^{\prime}} \delta Z_{a^{\prime} a}^{*} A_{i}^{R}\left(\tilde{t}_{a^{\prime}}\right)\left(\frac{1}{u-m_{\tilde{t}_{a}}^{2}}\right)+\delta A_{i}^{R}\left(\tilde{t}_{a}\right)\left(\frac{1}{u-m_{\tilde{t}_{a}}^{2}}\right) \\
& \left.+\frac{1}{2} \sum_{j} \delta \chi_{j i}^{L} A_{j}^{L}\left(\tilde{t}_{a}\right)\left(\frac{1}{u-m_{\tilde{t}_{a}}^{2}}\right)\right\}
\end{aligned}
$$

and from $\tilde{t}_{a}$ s.e.:

$$
N_{2 \eta}^{\text {s.e. } u}=-2 g_{s}\left(\frac{\lambda^{l}}{2}\right) \frac{1}{u-m_{a}^{2}} \sum_{a^{\prime}} \bar{v}_{c}\left(\chi_{i}^{-}\right)\left[A_{i}^{L}\left(\tilde{t}_{a^{\prime}}\right) P_{L}+A_{i}^{R}\left(\tilde{t}_{a^{\prime}}\right) P_{R}\right] u(b) \frac{\hat{\Sigma}_{a^{\prime} a}(u)}{u-m_{a^{\prime}}^{2}}
$$

The renormalized self-energy $\hat{\Sigma}_{a^{\prime} a}(u)$ is defined below. Following [20, 21, 22, 23] we have:

$$
\delta Z_{b a}=\frac{2 \Sigma_{b a}\left(m_{a}^{2}\right)}{m_{b}^{2}-m_{a}^{2}} \quad \delta Z_{a a}=-\left[\frac{d \Sigma_{a a}\left(p^{2}\right)}{d p^{2}}\right]_{p^{2}=m_{a}^{2}}
$$

These results allow to write the renormalized stop self-energies as:

$$
\hat{\Sigma}_{a a}\left(p^{2}\right)=\Sigma_{a a}\left(p^{2}\right)-\Sigma_{a a}\left(m_{a}^{2}\right)-\left(p^{2}-m_{a}^{2}\right)\left[\frac{d \Sigma_{a a}\left(p^{2}\right)}{d p^{2}}\right]_{p^{2}=m_{a}^{2}}
$$

and for $a \neq b$

$$
\hat{\Sigma}_{b a}\left(p^{2}\right)=\Sigma_{b a}\left(p^{2}\right)+\frac{p^{2}-m_{b}^{2}}{m_{\tilde{t}_{b}}^{2}-m_{a}^{2}} \Sigma_{b a}\left(m_{\tilde{t}_{a}}^{2}\right)+\frac{p^{2}-m_{a}^{2}}{m_{a}^{2}-m_{b}^{2}} \Sigma_{a b}^{*}\left(m_{b}^{2}\right)
$$

The renormalization condition on the mixing angle is defined [22] in order to ensure the finiteness of the squark vertices.

$$
\delta \theta_{t}=\frac{\Sigma_{12}\left(m_{1}^{2}\right)+\Sigma_{21}\left(m_{2}^{2}\right)}{2\left(m_{1}^{2}-m_{2}^{2}\right)}=\frac{1}{4}\left[\delta Z_{12}-\delta Z_{21}\right]
$$

which gives the needed:

$$
\delta R_{1 L}=\delta R_{2 R}=\delta \cos \theta_{t}=-\sin \theta_{t} \delta \theta_{t} \quad \delta R_{1 R}=-\delta R_{2 L}=\delta \sin \theta_{t}=\cos \theta_{t} \delta \theta_{t}
$$

The various cunter terms for the quarks and gauge bosons have the following explicit form in terms of self-energies; for b, t quark and gauge part:

$$
\begin{gathered}
\delta Z_{L}^{b}=\delta Z_{L}^{t} \equiv \delta Z_{L}=-\Sigma_{L}^{b}\left(m_{b}^{2}\right)-m_{b}^{2}\left[\Sigma_{L}^{\prime}\left(m_{b}^{2}\right)+\Sigma_{R}^{\prime b}\left(m_{b}^{2}\right)+2 \Sigma_{S}^{\prime b}\left(m_{b}^{2}\right)\right] \\
\delta Z_{R}^{b}=-\Sigma_{R}^{b}\left(m_{b}^{2}\right)-m_{b}^{2}\left[\Sigma_{L}^{\prime b}\left(m_{b}^{2}\right)+\Sigma_{R}^{\prime b}\left(m_{b}^{2}\right)+2 \Sigma_{S}^{\prime b}\left(m_{b}^{2}\right)\right] \\
\delta m_{b}=\frac{m_{b}}{2} \operatorname{Re}\left[\Sigma_{L}^{b}\left(m_{b}^{2}\right)+\Sigma_{R}^{b}\left(m_{b}^{2}\right)+2 \Sigma_{S}^{b}\left(m_{b}^{2}\right)\right]
\end{gathered}
$$




$$
\begin{gathered}
\delta Z_{1}^{W}-\delta Z_{2}^{W}=\frac{\Sigma^{\gamma Z}(0)}{s_{W} c_{W} M_{Z}^{2}} \\
\delta Z_{2}^{W}=-\Sigma^{\prime} \gamma \gamma(0)+2 \frac{c_{W}}{s_{W} M_{Z}^{2}} \Sigma^{\gamma Z}(0)+\frac{c_{W}^{2}}{s_{W}^{2}}\left[\frac{\delta M_{Z}^{2}}{M_{Z}^{2}}-\frac{\delta M_{W}^{2}}{M_{W}^{2}}\right] \\
\delta M_{W}^{2}=R e \Sigma^{W W}\left(M_{W}^{2}\right) \quad \delta M_{Z}^{2}=\operatorname{Re} \Sigma^{Z Z}\left(M_{Z}^{2}\right)
\end{gathered}
$$

while the couterterms for the gauge coupling lead to:

$$
\begin{gathered}
\frac{\delta g}{g}=\delta Z_{1}^{W}-\frac{3}{2} \delta Z_{2}^{W} \\
\frac{\delta A_{i}^{L}\left(\tilde{t}_{a}\right)}{A_{i}^{L}\left(\tilde{t}_{a}\right)}=\frac{1}{A_{i}^{L}\left(\tilde{t}_{a}\right)}\left\{\delta R_{a L} A_{i}^{L}\left(\tilde{t}_{L}\right)+R_{a L} \delta A_{i}^{L}\left(\tilde{t}_{L}\right)+\delta R_{a R} A_{i}^{L}\left(\tilde{t}_{R}\right)+R_{a R} \delta A_{i}^{L}\left(\tilde{t}_{R}\right)\right\} \\
\frac{\delta A_{i}^{R}\left(\tilde{t}_{a}\right)}{A_{i}^{R}\left(\tilde{t}_{a}\right)}=\frac{\delta R_{a L}}{R_{a L}}+\frac{\delta A_{i}^{R}\left(\tilde{t}_{L}\right)}{A_{i}^{R}\left(\tilde{t}_{L}\right)} \\
\frac{\delta A_{i}^{L}\left(\tilde{t}_{L}\right)}{A_{i}^{L}\left(\tilde{t}_{L}\right)}=\frac{\delta g}{g}+\frac{\delta Z_{1 i}^{+}}{Z_{1 i}^{+}} \\
\frac{\delta g}{A_{i}^{L}\left(\tilde{t}_{R}\right)}+\frac{\delta Z_{2 i}^{+}}{Z_{2 i}^{+}}+\frac{\delta m_{t}}{m_{t}}-\frac{\delta M_{W}}{M_{W}}-\frac{\delta \sin \beta}{\sin \beta} \\
A_{i}^{R}\left(\tilde{t}_{L}\right) \\
=\frac{\delta g}{g}+\frac{\delta Z_{2 i}^{-*}}{Z_{2 i}^{-*}}+\frac{\delta m_{b}}{m_{b}}-\frac{\delta M_{W}}{M_{W}}-\frac{\delta \cos \beta}{\cos \beta}
\end{gathered}
$$

From [24] we have for $\frac{\delta \tan \beta}{\tan \beta}$ :

$$
\begin{gathered}
\frac{\delta \tan \beta}{\tan \beta}=\frac{\operatorname{Re} \Sigma_{H^{+} W^{+}}\left(m_{H^{+}}^{2}\right)}{M_{W} \sin 2 \beta} \\
\frac{\delta \sin \beta}{\sin \beta}=\cos ^{2} \beta \frac{\delta \tan \beta}{\tan \beta} \quad \frac{\delta \cos \beta}{\cos \beta}=-\sin ^{2} \beta \frac{\delta \tan \beta}{\tan \beta}
\end{gathered}
$$

We need also the counterterms for the chargino mixing matrices. Applying the method of [22] 24] requiring the cancellation of the antihermitean part of the wave function renormalization, we have:

$$
\begin{aligned}
\delta Z_{1 i}^{+} & =\frac{1}{4} \sum_{k} Z_{1 k}^{+}\left(\delta \chi_{i k}^{L *}-\delta \chi_{k i}^{L}\right) \\
\delta Z_{2 i}^{+} & =\frac{1}{4} \sum_{k} Z_{2 k}^{+}\left(\delta \chi_{i k}^{L *}-\delta \chi_{k i}^{L}\right) \\
\delta Z_{2 i}^{-*} & =\frac{1}{4} \sum_{k} Z_{2 k}^{-*}\left(\delta \chi_{i k}^{R *}-\delta \chi_{k i}^{R}\right)
\end{aligned}
$$


Using the $\chi^{+}$chargino c.t. and s.e. $\delta \chi_{i j}^{L, R}$ listed below the counter terms for $\chi_{i}^{+}$are obtained from the field transformation:

$$
\chi_{i}^{+} \rightarrow\left(1+\frac{1}{2}\left[\delta \chi_{i j}^{L} P_{L}+\delta \chi_{i j}^{R} P_{R}\right]\right) \chi_{j}^{+}
$$

They are obtained by applying the method proposed in [24] [25] [26] and in terms of the $j \rightarrow i$ bubble of momentum $p$ they read:

$$
\Sigma_{i j}=\not p P_{L} \Sigma_{i j}^{L}+\not p P_{R} \Sigma_{i j}^{R}+P_{L} \Sigma_{i j}^{S}+P_{R} \Sigma_{i j}^{\bar{S}}
$$

with $\Sigma_{i j}^{\bar{S}}=\sum_{j i}^{S *}$ and:

$$
\begin{aligned}
& \delta \chi_{i i}^{L}=-\left\{\Sigma_{i i}^{L}\left(M_{i}^{2}\right)+M_{i}^{2}\left[\Sigma_{i i}^{\prime L}\left(M_{i}^{2}\right)+\Sigma_{i i}^{\prime R}\left(M_{i}^{2}\right)\right]+M_{i}\left[\Sigma_{i i}^{\prime S}\left(M_{i}^{2}\right)+\Sigma_{i i}^{\prime \bar{S}}\left(M_{i}^{2}\right)\right]\right\} \\
& \delta \chi_{i i}^{R}=-\left\{\Sigma_{i i}^{R}\left(M_{i}^{2}\right)+M_{i}^{2}\left[\Sigma_{i i}^{\prime L}\left(M_{i}^{2}\right)+\Sigma_{i i}^{\prime R}\left(M_{i}^{2}\right)\right]+M_{i}\left[\Sigma_{i i}^{\prime} S\left(M_{i}^{2}\right)+\Sigma_{i i}^{\prime} \bar{S}\left(M_{i}^{2}\right)\right]\right\}
\end{aligned}
$$

and for $i \neq j$

$$
\begin{aligned}
& \delta \chi_{i j}^{L}=\frac{2}{M_{i}^{2}-M_{j}^{2}}\left\{M_{j}^{2} \Sigma_{i j}^{L}\left(M_{j}^{2}\right)+M_{i} M_{j} \Sigma_{i j}^{R}\left(M_{j}^{2}\right)+M_{i} \Sigma_{i j}^{S}\left(M_{j}^{2}\right)+M_{j} \Sigma_{i j}^{\bar{S}}\left(M_{j}^{2}\right)\right\} \\
& \delta \chi_{i j}^{R}=\frac{2}{M_{i}^{2}-M_{j}^{2}}\left\{M_{j}^{2} \Sigma_{i j}^{R}\left(M_{j}^{2}\right)+M_{i} M_{j} \Sigma_{i j}^{L}\left(M_{j}^{2}\right)+M_{j} \Sigma_{i j}^{S}\left(M_{j}^{2}\right)+M_{i} \Sigma_{i j}^{\bar{S}}\left(M_{j}^{2}\right)\right\}
\end{aligned}
$$

All the self-energy functions $\Sigma$ are computed from the diagrams of Fig. 3 .

[1] M. Beccaria, G. Macorini, L. Panizzi, F. M. Renard and C. Verzegnassi, Phys. Rev. D 74 (2006) 093009 arXiv:hep-ph/0610075.

[2] G. Bozzi, B. Fuks, B. Herrmann and M. Klasen, Nucl. Phys. B 787 (2007) 1 arXiv:0704.1826 [hep-ph].

[3] L. G. Jin, C. S. Li and J. J. Liu, Eur. Phys. J. C 30 (2003) 77 arXiv:hep-ph/0210362.

L. G. Jin, C. S. Li and J. J. Liu, Phys. Lett. B 561 (2003) 135 arXiv:hep-ph/0307390.

[4] M. Beccaria, F. M. Renard and C. Verzegnassi, arXiv:hep-ph/0203254.

[5] M. Beccaria, G. Macorini, F. M. Renard and C. Verzegnassi, Phys. Rev. D 73 (2006) 093001 arXiv:hep-ph/0601175.

[6] M. Beccaria, F. M. Renard and C. Verzegnassi, Phys. Rev. D 71 (2005) 033005 arXiv:hep-ph/0410089. 
[7] J. Kublbeck, M. Bohm and A. Denner, Comput. Phys. Commun. 60, 165 (1990).

T. Hahn, Comput. Phys. Commun. 140, 418 (2001) arXiv:hep-ph/0012260].

T. Hahn and C. Schappacher, Comput. Phys. Commun. 143, 54 (2002) arXiv:hep-ph/0105349.

[8] T. Hahn and M. Perez-Victoria, Comput. Phys. Commun. 118, 153 (1999) arXiv:hep-ph/9807565.

T. Hahn and M. Rauch, Nucl. Phys. Proc. Suppl. 157, 236 (2006) arXiv:hep-ph/0601248.

[9] U. Baur, S. Keller and D. Wackeroth, Phys. Rev. D 59, 013002 (1999) arXiv:hep-ph/9807417.

[10] A. D. Martin, R. G. Roberts, W. J. Stirling and R. S. Thorne, Eur. Phys. J. C 39, 155 (2005) arXiv:hep-ph/0411040.

[11] J. H. Kuhn, A. Kulesza, S. Pozzorini and M. Schulze, Nucl. Phys. B 797, 27 (2008) arXiv:0708.0476 [hep-ph]].

[12] J. Pumplin, D. R. Stump, J. Huston, H. L. Lai, P. M. Nadolsky and W. K. Tung, JHEP 0207, 012 (2002) arXiv:hep-ph/0201195.

[13] M. Roth and S. Weinzierl, Phys. Lett. B 590, 190 (2004) arXiv:hep-ph/0403200.

[14] M. Beccaria, C. M. Carloni Calame, G. Macorini, G. Montagna, F. Piccinini, F. M. Renard and C. Verzegnassi, Eur. Phys. J. C 53 (2008) 257 [arXiv:0705.3101 [hep-ph]].

[15] M. Beccaria, C. M. Carloni Calame, G. Macorini, E. Mirabella, F. Piccinini, F. M. Renard and C. Verzegnassi, Phys. Rev. D 77 (2008) 113018 [arXiv:0802.1994 [hep-ph].

[16] M. Beccaria, G. Macorini, F. M. Renard and C. Verzegnassi, Phys. Rev. D 74, 013008 (2006) arXiv:hep-ph/0605108.

[17] ATLAS Data Challenge 2 DC2 points: http://paige.home.cern.ch/paige/fullsusy/romeindex.html.

[18] B. C. Allanach et al., The Snowmass points and slopes: Benchmarks for SUSY searches, in Proc. of the APS/DPF/DPB Summer Study on the Future of Particle Physics (Snowmass 2001) ed. N. Graf, In the Proceedings of APS / DPF / DPB Summer Study on the Future of Particle Physics (Snowmass 2001), Snowmass, Colorado, 30 Jun - 21 Jul 2001, pp P125 arXiv:hep-ph/0202233.

[19] B. Clément, ATL-SLIDE-2008-117; CERN-ATL-SLIDE-2008-117.

[20] A. Djouadi, W. Hollik and C. Junger, Phys. Rev. D 55 (1997) 6975 arXiv:hep-ph/9609419].

[21] S. Kraml, H. Eberl, A. Bartl, W. Majerotto and W. Porod, Phys. Lett. B 386 (1996) 175 
arXiv:hep-ph/9605412.

[22] H. Eberl, A. Bartl and W. Majerotto, Nucl. Phys. B 472 (1996) 481 arXiv:hep-ph/9603206.

[23] A. Arhrib and W. Hollik, JHEP 0404 (2004) 073 arXiv:hep-ph/0311149.

[24] L. H. Wan, W. G. Ma, R. Y. Zhang and Y. Jiang, Phys. Rev. D 64 (2001) 115004 arXiv:hep-ph/0107089.

[25] A. Denner and T. Sack, Nucl. Phys. B 347 (1990) 203.

[26] B. A. Kniehl and A. Pilaftsis, Nucl. Phys. B 474, 286 (1996) arXiv:hep-ph/9601390. 


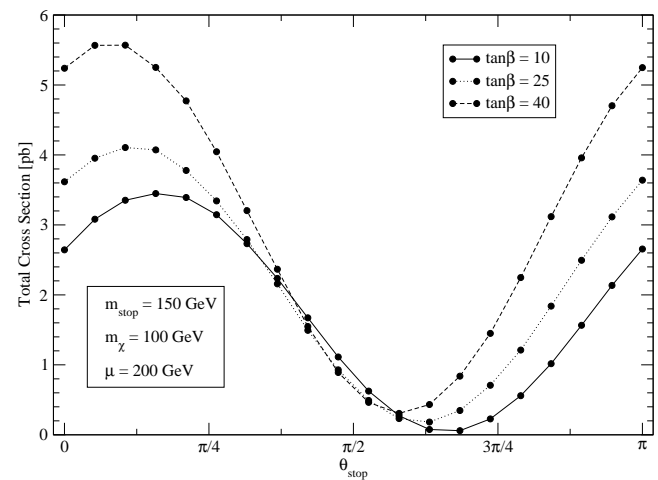

(a)

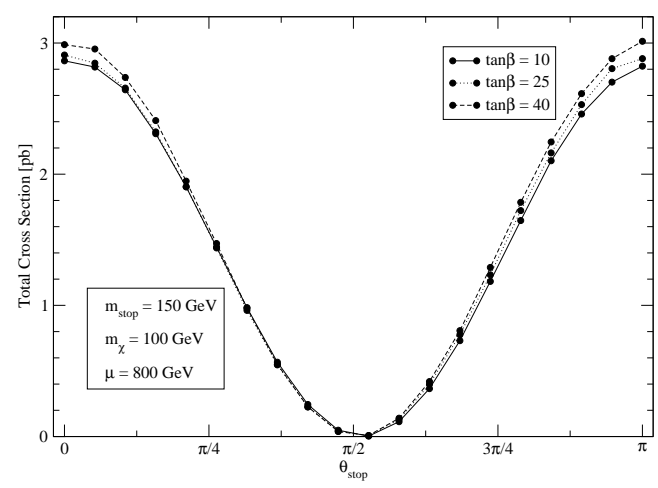

(c)

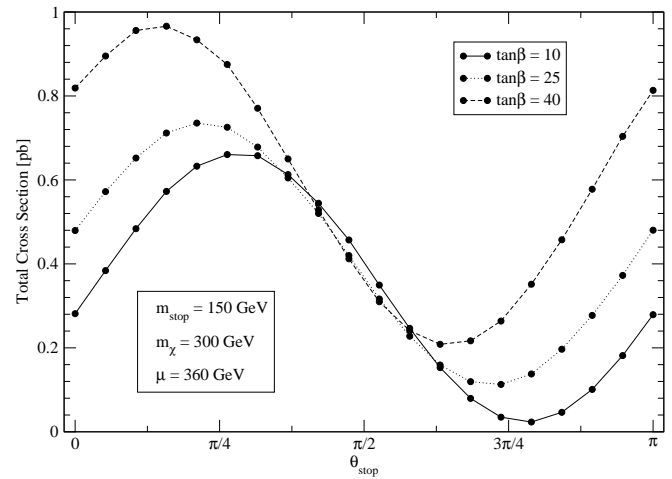

(b)

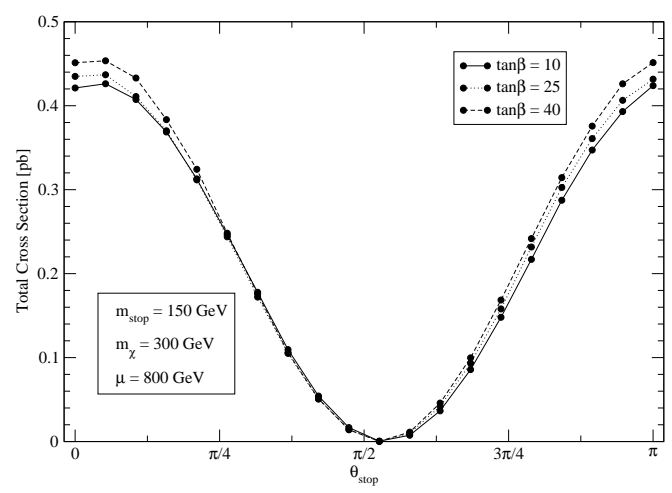

(d)

FIG. 1: Parameter dependence of the total cross section at Born level 


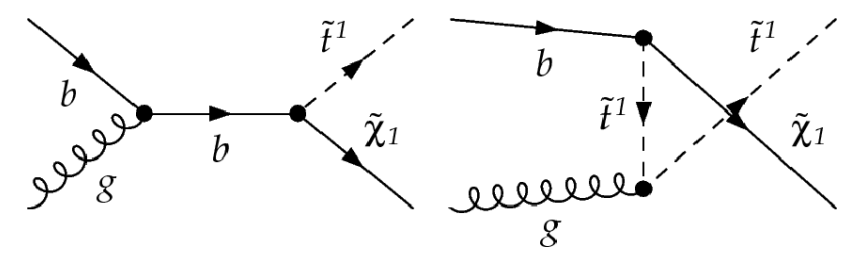

1

2

FIG. 2: Born Diagrams: s-channel bottom exchange and u-channel stop exchange
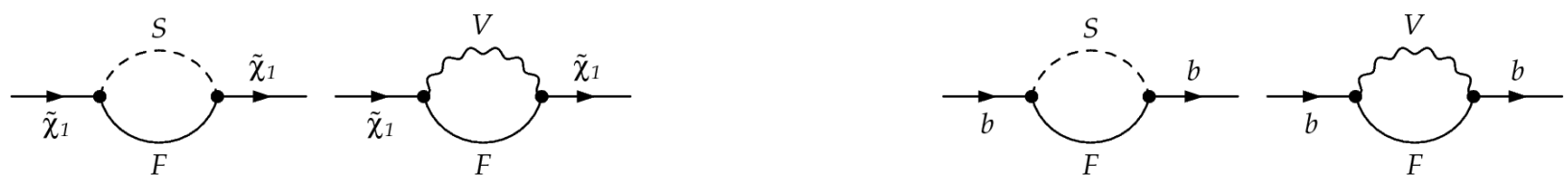

1

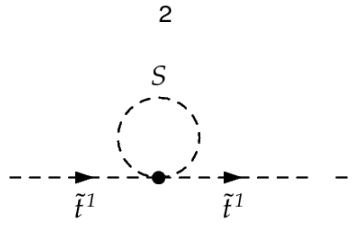

1

2

1

2

3

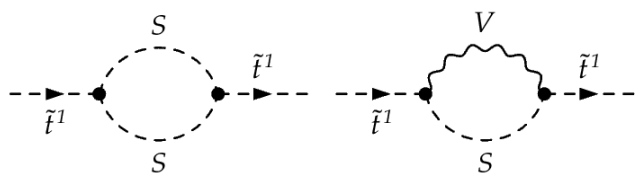

4

5

FIG. 3: Self-energy diagrams for chargino, bottom, and stop lines 

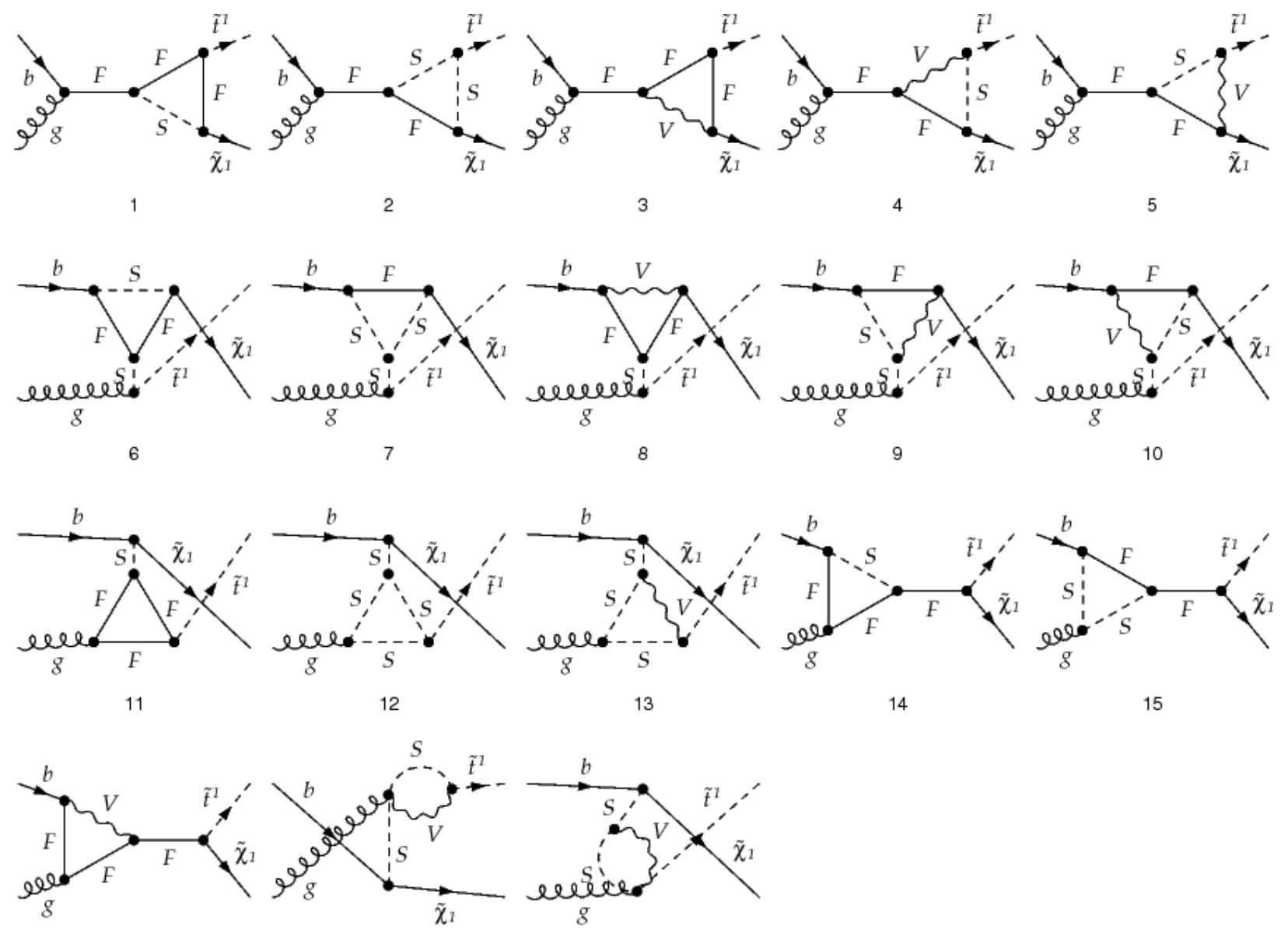

16

17

18

FIG. 4: Triangle and 4-leg diagrams 

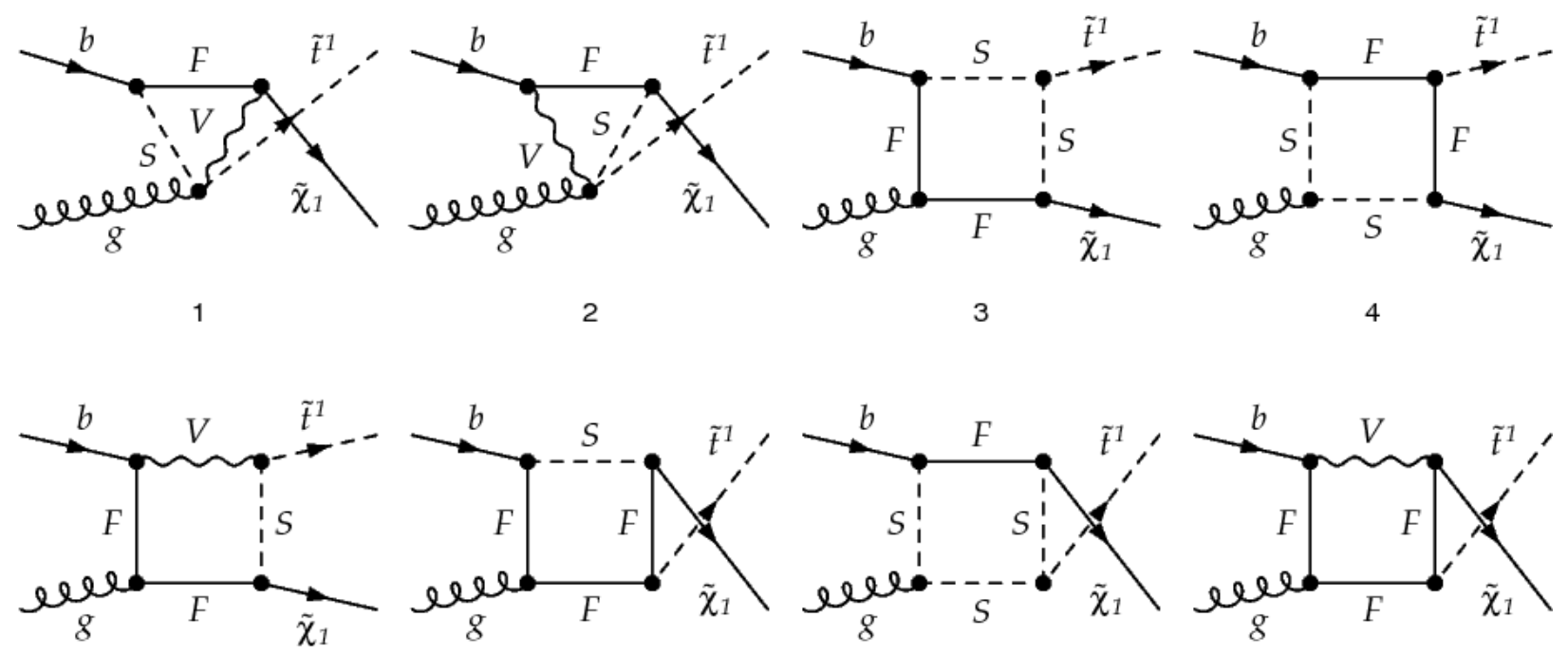

5

6

7

8

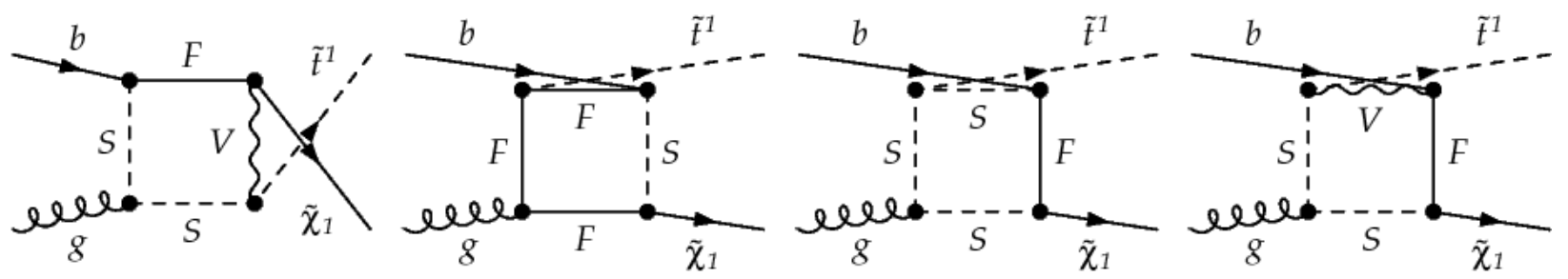

9

10

11

12

FIG. 5: Triangle and box diagrams 


\section{LS1: Energy Dependence}

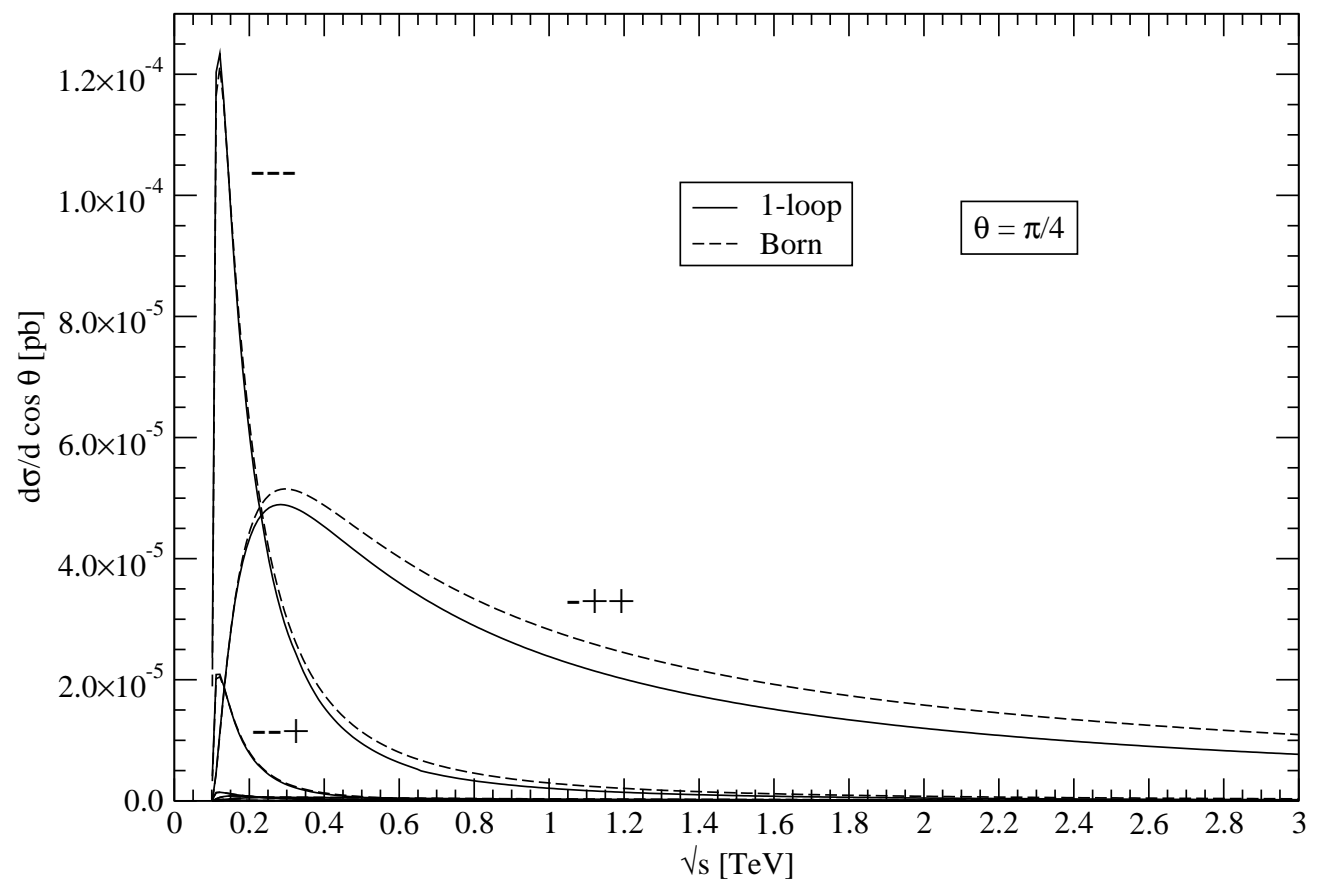

LS2: Energy Dependence

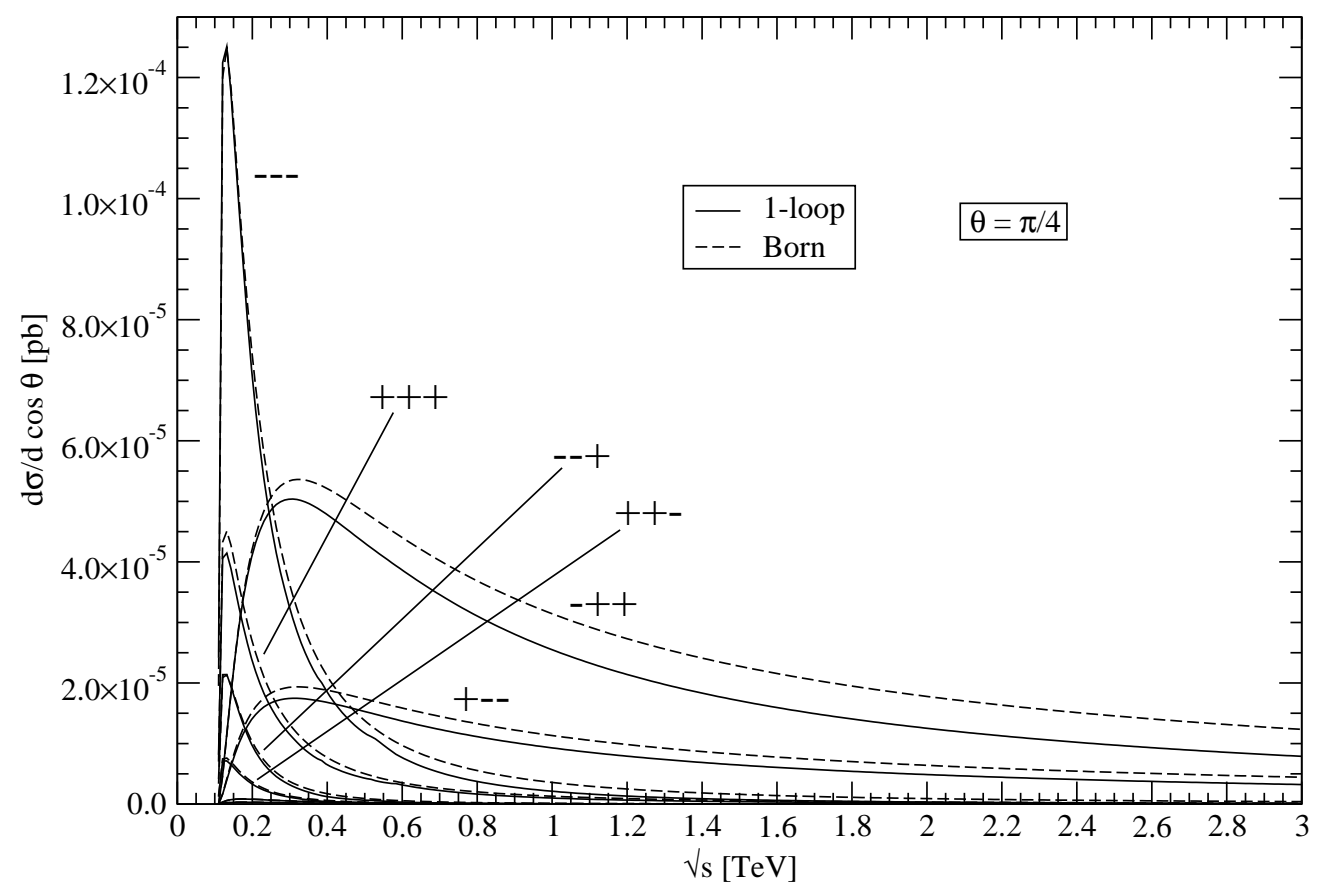

FIG. 6: Energy dependence for leading helicity amplitudes in LS1 and LS2. 

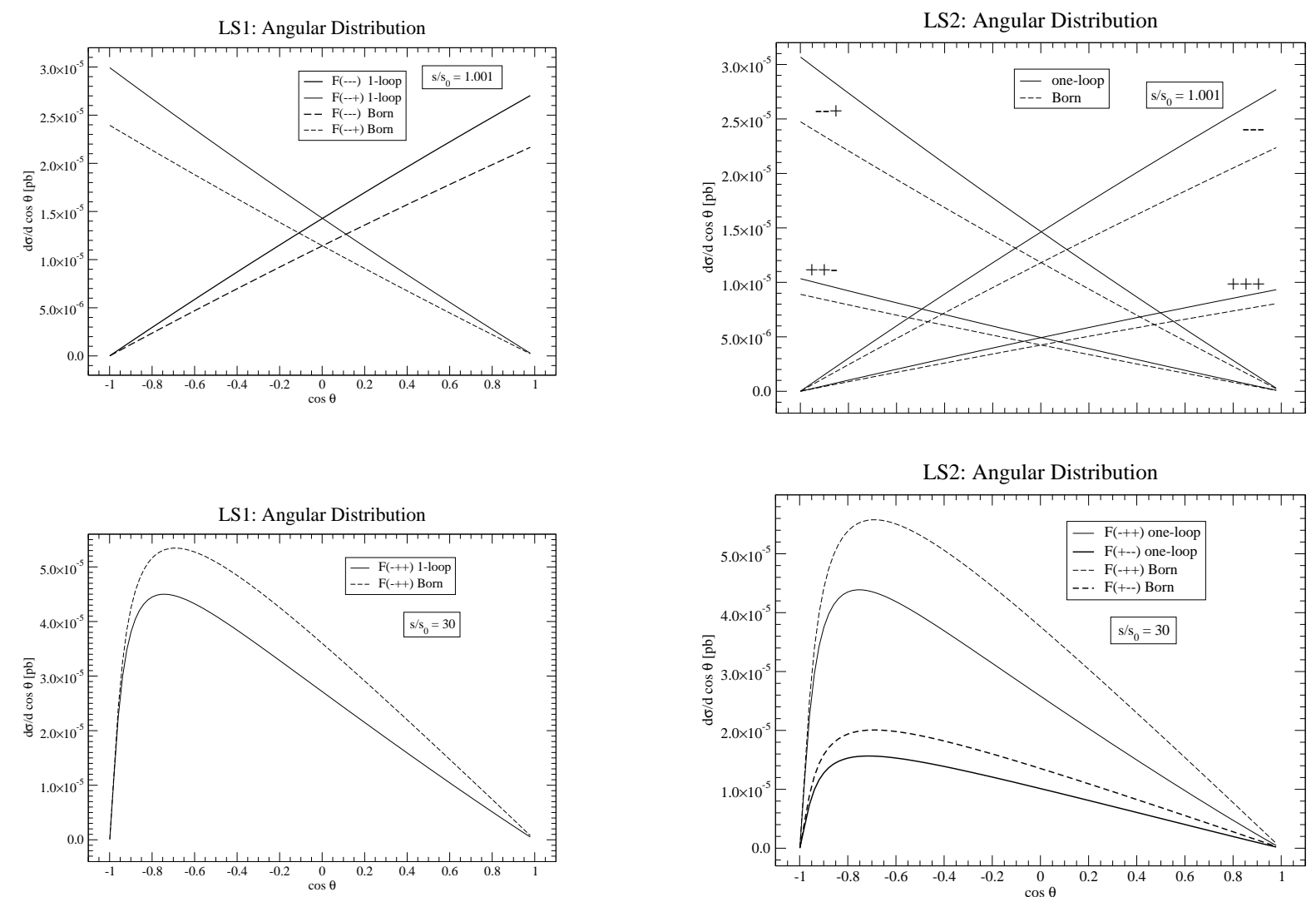

FIG. 7: Angular distribution for leading helicity amplitudes in LS1 and LS2 in the low and high energy limits. 

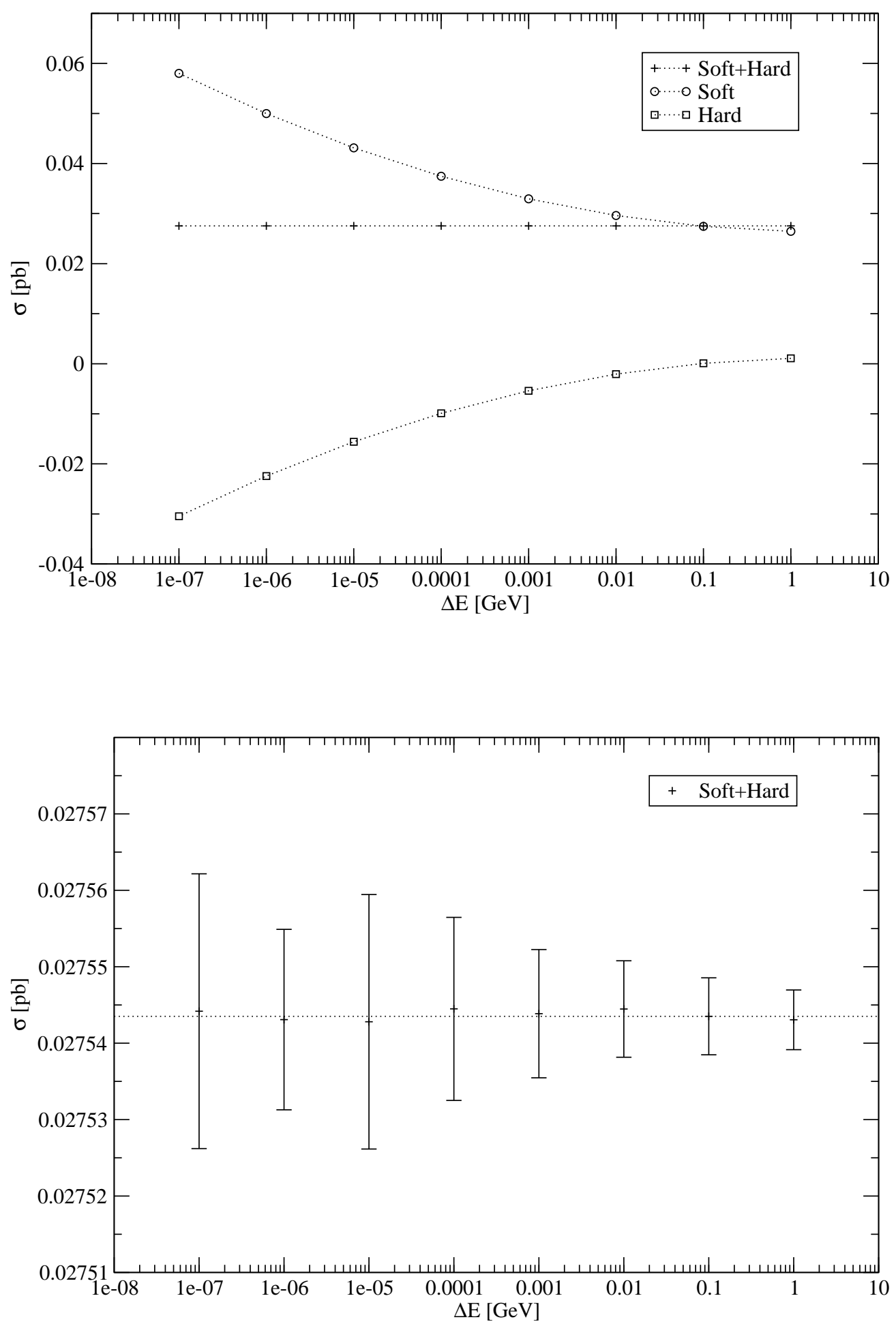

FIG. 8: Upper panel: dependence of the $\mathcal{O}(\alpha)$ soft plus virtual and hard cross sections on the soft-hard separator $\Delta E$. Lower panel: independence of the sum of $\mathcal{O}(\alpha)$ soft plus virtual and hard cross sections of the separator $\Delta E$. 


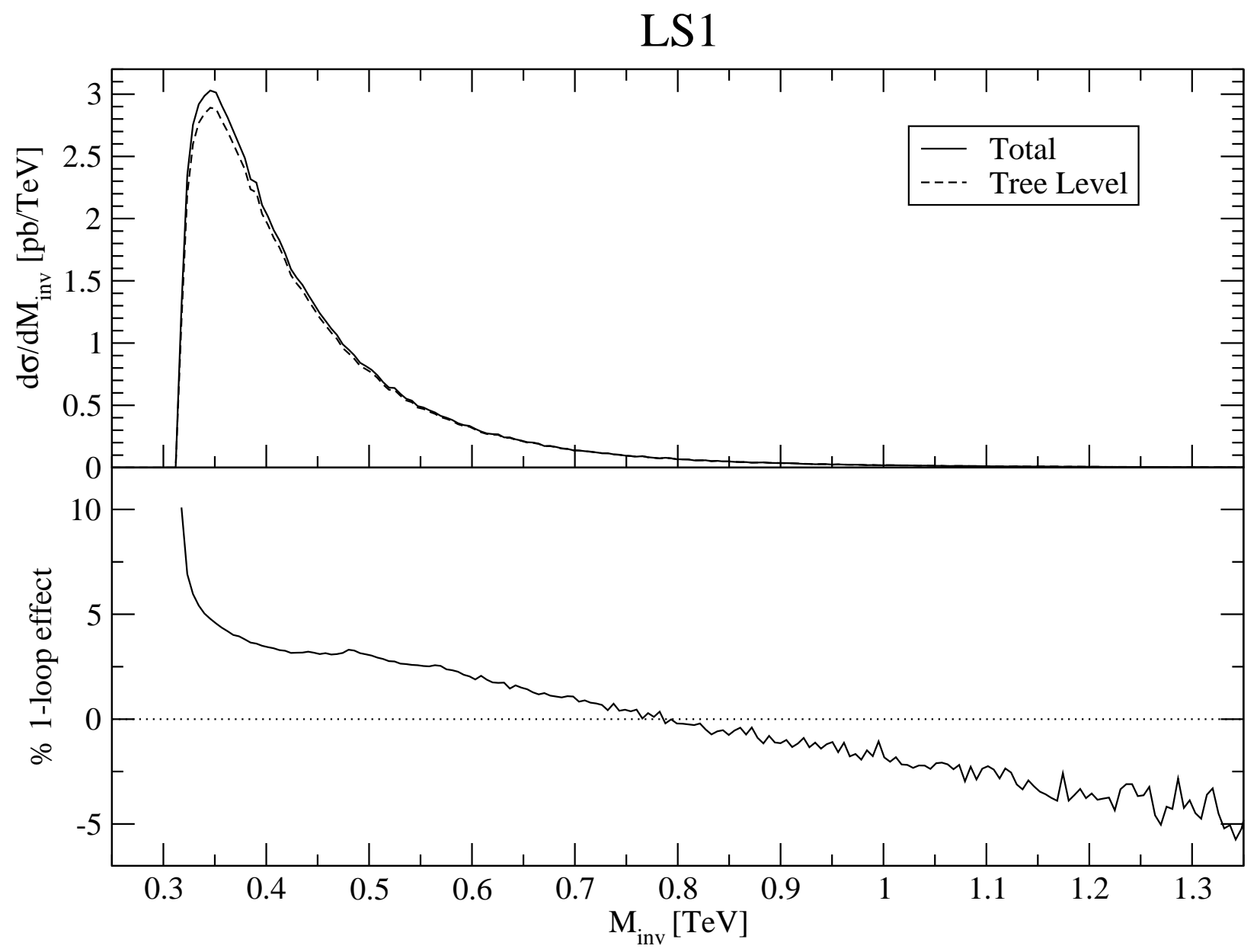

FIG. 9: Differential distribution (upper panel) and percentage one-loop effect in the LS1 point; $m_{\tilde{t}_{1}}=214.5 \mathrm{GeV}, m_{\chi_{1}}=103.6 \mathrm{GeV}$. 


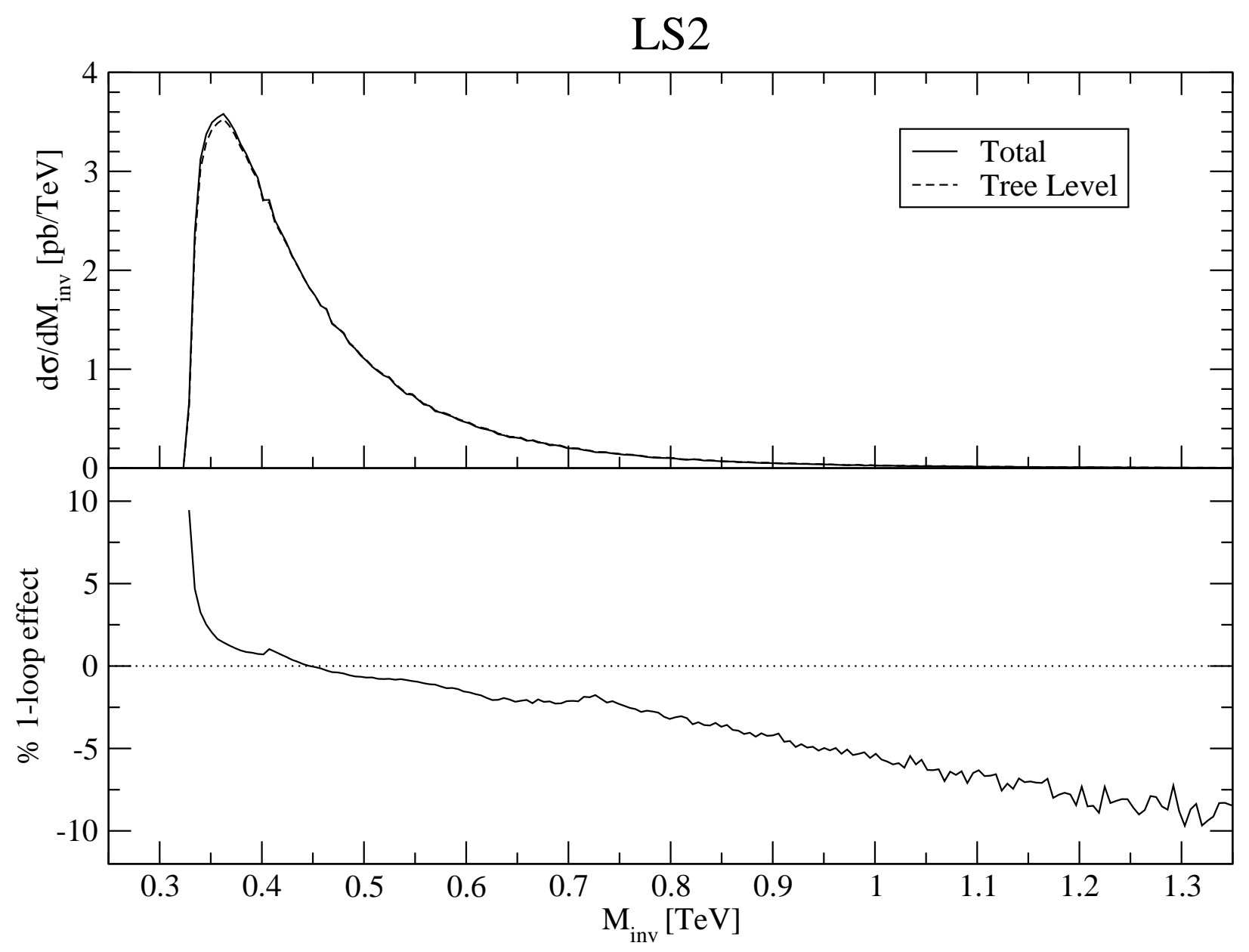

FIG. 10: Differential distribution (upper panel) and percentage one-loop effect in the LS2 point; $m_{\tilde{t}_{1}}=224.6 \mathrm{GeV}, m_{\chi_{1}}=106.9 \mathrm{GeV}$. 